\title{
O Uso de Projetos-Piloto para Avaliação da Efetividade da Melhoria de Processos
}

\author{
Reinaldo C. Silva Filho, Ana Regina C. Rocha, Guilherme H. Travassos \\ Programa de Engenharia de Sistemas e Computação - COPPE/UFRJ \\ Caixa Postal 68.511 - CEP 21945-970 - Rio de Janeiro - RJ - Brasil \\ \{cabral, darocha, ght\} @cos.ufrj.br
}

\begin{abstract}
This paper presents a framework to performing pilot projects on a systematic, planned and controlled way to allow that the effects of the software process change can be observed and measured before its institutionalization.
\end{abstract}

Resumo. Este artigo apresenta um arcabouço para a realização de projetospiloto de forma sistemática, planejada e controlada para que os efeitos das mudanças dos processos de software possam ser observados e mensurados antes de sua institucionalização.

\section{Introdução}

As organizações que fazem uso de processos como base para o desenvolvimento de seus produtos frequentemente se deparam com a necessidade de realizar alterações para adequá-los às especificidades do negócio e aos objetivos gerais da organização. A partir de avaliações e experiências bem conduzidas, as organizações podem identificar e adaptar boas práticas às suas necessidades e incorporá-las em seus processos. Medições de produto e processo podem ser usadas para avaliar a efetividade das melhorias. Comparações entre resultados de projetos-piloto e dados históricos, bem como tendências gerais podem testar e demonstrar os efeitos da mudança do processo [WALRAD e MOSS 1993].

Kelley e Morath (2001) ilustram três situações em iniciativas em prol da melhoria: (i) Uma mudança é realizada, porém nenhum dado foi coletado antes ou depois da mudança para avaliar se os efeitos foram positivos ou negativos; (ii) A mudança é realizada, mas os dados que caracterizam a situação anterior à mudança não são coletados; e (iii) Há dados coletados antes e depois da mudança que permitem verificar se os efeitos foram positivos ou não. Mesmo no terceiro caso, algumas questões precisam ser consideradas durante a análise dos efeitos da mudança: Os dados coletados estão corretos? Existem outros fatores que podem dificultar a interpretação dos resultados? Os efeitos observados podem ser generalizados? A mudança foi conduzida de forma correta a ponto de apresentar os reais efeitos? As pessoas foram devidamente preparadas para a mudança? A mudança poderia ser melhor? Desconsiderar estes aspectos significa estar suscetível a interpretações equivocadas, o que pode orientar a organização na direção errada.

Por outro lado, considerar todas estas questões não é uma tarefa trivial. Na academia, pesquisadores fazem uso do paradigma experimental para colher evidências e obter acurácia nas avaliações dos efeitos da aplicação de novas tecnologias [FENTON et 
al. 1994; ZELKOWITZ et al. 2003; DYBA et al. 2005]. Neste contexto, dois obstáculos oferecem resistência à transferência tecnológica dos achados da academia para a indústria: a ausência de controle nas experiências em ambiente industrial e a ausência de realismo na realização de experimentos controlados. Além disso, é necessário convencer a indústria sobre a validade e aplicabilidade dos resultados [SJØBERG et al. 2002].

Este trabalho propõe um arcabouço para a realização de projetos-piloto no ambiente industrial de forma sistemática, planejada e controlada para que os efeitos das melhorias em processos de software possam ser observados e mensurados adequadamente antes da institucionalização das mudanças.

Além desta seção introdutória, cinco seções compõem o artigo. As três seções subseqüentes tratam do conceito de piloto e a relação com modelos de qualidade, da avaliação da efetividade das propostas de melhoria, apresentando mecanismos úteis a este propósito e provendo uma discussão sobre a utilização de projetos-pilotos. A quinta seção apresenta um arcabouço para realização de pilotos e seus elementos constituintes. Por fim, a seção 6 provê notas conclusivas.

\section{Modelos e Normas em Prol da Qualidade e Projetos-Piloto}

Modelos e normas que tratam da melhoria de processos de software, tais como IDEAL [MCFEELEY 1996], QIP [BASILI et al. 1994], CMMI [CHRISSIS et al. 2003], MRMPS [MPS.BR 2005] e a ISO/IEC 15504-4 [ISO 2004], sugerem o uso de projetospiloto para avaliar a efetividade das ações em prol da melhoria quando há riscos e incertezas associadas.

O IDEAL sugere que o uso de pilotos seja na fase de ação, quando há necessidade de avaliar potenciais soluções de melhoria.

No QIP um projeto é utilizado como piloto no primeiro ciclo (controlado) durante a fase de execução e a partir dele são coletados indicadores para prevenir e resolver os problemas observados.

O CMMI sugere o uso de pilotos a partir do nível 3, nas áreas Foco no Processo Organizacional e Análise de Decisão e Resolução. Na área Foco no Processo Organizacional, mudanças importantes devem ser testadas por meio de pilotos antes de serem institucionalizadas. Em Análise de Decisão e Resolução, o piloto serve de instrumento para revelar problemas, avaliar diferentes critérios e alternativas que devem ser consideradas para anular um potencial viés na decisão. No nível 5, na área de Inovação e Implantação na Organização, pilotos são conduzidos para avaliar mudanças significativas envolvendo melhorias não testadas e de alto risco ou melhorias inovadoras, antes que elas sejam amplamente difundidas [CHRISSIS et al. 2003]. Essa área de processo também ratifica a necessidade de planejar o piloto, obter o comprometimento dos envolvidos, executá-lo em um ambiente comum aos demais projetos da organização, acompanhar a sua execução, verificá-lo com relação ao que foi planejado, revisar e documentar os seus resultados (melhorias, problemas encontrados e lições aprendidas).

O MR-MPS faz referência ao uso de pilotos no nível A para apoiar a tomada de decisão quanto à institucionalização das ações de melhoria. O uso de pilotos auxilia a análise e priorização das propostas de melhoria do processo e de melhoria tecnológica. $\mathrm{O}$ 
modelo afirma que os projetos-pilotos devem ser planejados e os resultados e lições aprendidas registradas.

A ISO/IEC 15504-4 menciona o uso de pilotos no contexto dos programas de melhoria, na avaliação das propostas obtidas a partir da análise das oportunidades de melhoria. A norma preconiza que o programa seja conduzido como um projeto que deve ser planejado, mensurado, monitorado e ter seus resultados avaliados.

A partir da menção feita ao termo "piloto" pelos modelos supracitados, observase uma visão compartilhada do conceito no contexto da melhoria de processos de software: o piloto é um instrumento utilizado para avaliar os efeitos da mudança em processos de software em um ambiente real.

Em alguns modelos, a maneira que as práticas são sugeridas não é explícita, portanto dependem da interpretação do leitor. A ISO/IEC 15504-4, por exemplo, associa o estabelecimento de métricas, planejamento e treinamento ao projeto do programa de melhoria como um todo, não especificamente para a realização de um piloto, que pode ou não estar contido no programa de melhoria. Portanto, subentende-se que todo piloto contido em um programa de melhoria deverá ser submetido a tais práticas.

Mesmo sugerindo práticas e recomendando o uso de pilotos em iniciativas de melhoria de processos, estes modelos não descrevem procedimentos que permitam avaliar de maneira consistente os efeitos obtidos a partir das mudanças dos processos.

\section{Instrumentos para Avaliação da Efetividade das Propostas de Melhoria}

Estudos experimentais, simulações e benchmarking são instrumentos que também podem apoiar a avaliação da efetividade das propostas de melhoria. A seguir será apresentada uma visão geral da utilidade destes instrumentos.

\subsection{Estudos Experimentais}

A condução de experimentos controlados é o método clássico para identificar relacionamentos causa-efeito [SJØBERG et al. 2005].

Kitchenham et al. (1995) defende que estudos de caso auxiliam a indústria a avaliar os benefícios de métodos e ferramentas, além de ser uma forma econômica e efetiva para assegurar que a mudança no processo propiciará os resultados desejados. Ela também provê orientações que permite identificar sobre quais condições os estudos de caso são mais adequados e fornece um guia para auxiliar a condução destes estudos de forma a embutir mais rigor no decorrer da investigação.

\subsection{Simulação}

A Simulação é um método controlado utilizado para avaliar os efeitos das mudanças e as relações entre as variáveis que estão sendo observadas [PFAHL e BIRK 2000; TVEDT e COLLOFELLO 1995].

As principais motivações para desenvolver ou utilizar um modelo de simulação residem no baixo custo, na limitação riscos e/ou no decremento do esforço logístico para manipular o sistema de interesse [KELLNER et al. 1999]. Neste sentido, iniciativas têm 
sido publicadas para demonstrar a aplicabilidade da simulação na melhoria de processos. Höst et al. (2001) utiliza a simulação de um processo de engenharia de requisitos para observar as condições de sobrecarga e encontrar mudanças que possam remover os gargalos do processo. Pfahl e Lebsanft (2000) também aplicam simulação no processo de engenharia de requisitos para analisar quanto esforço financeiro teria que ser investido para estabilizar e alcançar o ponto "ótimo" do processo. Raffo (1993) apresenta um exemplo onde potenciais mudanças do processo são quantitativamente avaliadas através de uma abordagem de simulação estocástica antes de comprometer recursos e esforços para implantação das mudanças. Além de tentar predizer o impacto da mudança realizada nos processos, a proposta de Raffo visa apoiar a priorização de ações de melhoria e avaliar o desempenho do modelo de processo atual com relação ao modelo planejado.

Ahmed-Nacer (2004) descreve uma abordagem para modificar modelos de processo e processos de software, que suporta diferentes estratégias de avaliação e permite a adaptação do processo "em vôo" (durante a execução). Durante a simulação, o modelo de processo de software é atualizado e validado através de múltiplas simulações antes de sua reexecução. $\mathrm{O}$ fato da abordagem não considerar o fator humano, bem como as diversas variáveis ambientais que podem influenciar a avaliação da mudança, restringe o uso do termo "validar" pelo o autor.

Outro aspecto não trivial envolvendo o uso de simulação é a modelagem de sistemas dinâmicos, que requer uma base de conhecimento contendo as relações entre fatores que influenciam o comportamento do modelo. Kellner et al. (1999) apontam questões importantes que precisam ser tratadas, a exemplo da identificação das variáveis de resposta e sua relação com o escopo do modelo e a definição dos parâmetros de entrada, que podem chegar a algumas centenas. Cada uma destas questões envolve um esforço e quantidade de conhecimento que dificilmente pequenas e médias empresas teriam condições de patrocinar.

Zelkowitz et al. (2003) afirma que a limitação da simulação reside na acurácia da representação dos modelos, ou seja, a simulação é conduzida em um ambiente artificial controlado que pode não apresentar todas as características que exercem influência nas atividades em um ambiente real. Esta limitação dificulta a observação dos efeitos reais das mudanças no ambiente industrial. Portanto, a simulação é útil para observar efeitos em um contexto limitado, levando em consideração que o mundo real é muito mais rico e exerce muitas influencias sobre os projetos. Münch e Armbrust (2003) apresentam uma breve, porém valiosa, discussão das limitações encontradas durante a experiência da construção de um modelo para simulação de processos de software.

Para suprimir óbices relacionados ao custo para construção dos modelos, a acurácia da representação e facilidades na interação, iniciativas como IMMoS ${ }^{1}$ [PFAHL e RUHE 2002] e VSEL ${ }^{2}$ [MÜNCH, PFAHL e RUS 2005] têm concentrado esforços

\footnotetext{
1 Integrated Measurement, Modeling, and Simulation. Método para mensuração, modelagem e simulação integrada de processos de software.

${ }^{2}$ Virtual Software Engineering Laboratory. O Laboratório Virtual de Engenharia de Software consiste em um conjunto de módulos de simulação de processos de software desenvolvido sistematicamente que podem ser combinados e adaptados para apoiar a solução de um problema, prover suporte à decisão e/ou analisar um conjunto de atividades dentro de um contexto específico.
} 
para viabilizar o amplo uso de simulação para avaliação da efetividade das propostas de melhoria em processos de software.

\subsection{Benchmarking}

Através de benchmarking as organizações observam outras empresas que realizam as práticas propostas como melhoria para organização e verificam os resultados obtidos por estas organizações a partir do uso destas práticas. O principal problema é o contexto organizacional, pois o sucesso da adoção de uma prática ou tecnologia em determinadas organizações não garante o mesmo sucesso em outras. Além disso, é importante determinar o que faz determinadas práticas passíveis de comparação.

Beitz e Wieczorek (2000) ilustram como utilizar benchmarking no contexto de programas de melhoria e a aplicação da técnica OSR (Optimised Set Reduction) para viabilizar a comparação dos dados coletados a partir dos projetos. Card e Zubrow (2001) abordam aspectos associados com o benchmarking quantitativo e fatores críticos de sucesso na realização do benchmarking.

\subsection{Combinação de Abordagens.}

Para cada tipo de iniciativa de melhoria existem métodos que podem ser combinados para avaliar a efetividade das mudanças propostas.

Por exemplo, em uma iniciativa que envolve a utilização processos inovadores, utilizar um projeto-piloto para avaliar um processo de desenvolvimento completamente novo, sem antes avaliar as chances de sucesso é perigoso. Nessas ocasiões, antes de investir esforço em um estudo mais elaborado, é recomendado combinar a realização de benchmarking, entrevistas e avaliações de especialistas para evitar desgastes desnecessários.

Na melhoria contínua, mais baseada em passos pequenos e evolucionários do que inovações revolucionárias [PAULK et al. 1993], o uso de pilotos pode ser útil, visto que o escopo das mudanças é mais limitado e, consequentemente, o dimensionamento dos riscos é facilitado. Com o objetivo de avaliar o impacto da inclusão da revisão de código na Ericsson Software Technology AB, Höst e Johansson (2000) combinaram a realização de entrevistas à execução de um experimento para avaliar o desempenho de dois métodos de revisão de código antes de institucionalizar as mudanças no processo.

Outra forma de combinar os mecanismos de avaliação é utilizar a simulação para priorizar as alterações no processo, o benchmarking para estabelecer um baseline e o estudo de caso para avaliar a efetividade da proposta com relação ao baseline.

\section{O uso de pilotos na avaliação das propostas de melhoria}

A execução de projetos pilotos pode servir a vários propósitos, dentre eles: (i) Identificar questões relacionadas ao conhecimento tácito [SHULL et al. 2004], tornando-o explícito à medida que o piloto é executado, servindo como uma importante fonte de lições aprendidas; (ii) Avaliar a conformidade do processo antes que qualquer esforço significante de replicação seja realizado [SHULL et al. 2004]; e (iii) Avaliar mudanças significativas ou melhorias inovadoras que não foram experimentadas e possuem alto risco, antes de serem amplamente disseminadas na organização [CHRISSIS et al. 2003]. 
O uso de pilotos também pode apoiar de forma significativa a diminuição do tempo para institucionalização das melhorias, a exemplo da empresa Silicon and Software Systems, que reduziu o tempo médio para avaliar e introduzir mudança dos processos de 533 dias para menos que 112 dias através do uso gerenciado de projetospiloto [O'HARA 2000].

Fowler e Rifkin (1990) afirmam que a instalação de novos procedimentos e tecnologias que apóiam a melhoria de processos é uma atividade contínua e de longo prazo e que estas instalações devem ser iniciadas com uma instalação piloto. "Pilotos são essenciais quando a organização não tem experiência com a tecnologia ou a tecnologia será aplicada em um novo domínio com uma equipe inexperiente." [Ibidem, p. 51, tradução nossa]. Os autores tratam a realização de um piloto como um experimento controlado e sugerem procedimentos para realização de pilotos que envolvem a utilização de novas tecnologias.

Os resultados negativos de um projeto piloto planejado e executado adequadamente deve ser encarado de uma forma positiva, pois o principal resultado nestes casos é a identificação de uma "pseudo melhoria" que não traria benefícios e consumiria esforço e investimento para ser implantada na organização. Além disso, a experiência provê um valioso conhecimento a respeito dos aspectos observados no piloto.

\section{Um arcabouço para realização de pilotos}

À primeira vista, a realização de um piloto consiste em aplicar a proposta de melhoria em um projeto e verificar seus resultados. Porém, há um conjunto de questões a serem consideradas para se executar um piloto e obter acurácia na avaliação da proposta. Dentre elas: (i) $\mathrm{O}$ projeto selecionado reflete a realidade dos demais projetos da organização que serão contemplados com as ações que estão sendo testadas? (ii) Um projeto é suficiente para avaliar a proposta? (iii) As pessoas precisam ser treinadas? Em que? (iv) Qual aspecto do projeto que está associado à proposta a ser testada? Como estes aspectos serão mensurados? (v) Como os resultados do projeto conduzirão a avaliação da efetividade da proposta de melhoria? (vi) O que se espera do projeto para afirmar se a proposta é aplicável ou não a organização? (vii) Que fatores podem interferir na condução do projeto e afetar a avaliação da proposta de melhoria? (viii) O que pode ser feito para minimizar estas influencias? (ix) A proposta de melhoria pode ser refinada?

Com o objetivo de tratar estas questões, foi construído um arcabouço, baseado em uma abordagem experimental, para realização de pilotos em prol da avaliação da efetividade das propostas de melhorias no âmbito industrial.

Conduzir um projeto-piloto capaz de testar as ações de melhoria sem interferir na logística e nos aspectos relacionadas a custo, disponibilidade de pessoal e infra-estrutura é um desafio, pois a inclusão de novas atividades em um projeto tem impacto direto no cronograma e na alocação de recursos. A proposta foi concebida com o intuito de minimizar a influência destes fatores e, ao mesmo tempo, potencializar os resultados obtidos na avaliação da efetividade das melhorias.

O arcabouço é constituído por três elementos: um processo, que orienta as ações a serem executadas durante a realização do piloto; uma ferramenta, que apóia a 
execução das atividades do processo; e um guia, que contém conhecimento sobre o processo e sobre a ferramenta e serve de roteiro para permitir a utilização eficiente do arcabouço.

\subsection{O Elemento Processo}

O modelo de processo foi o primeiro elemento a ser concebido e foi influenciado pelos trabalhos Kitchenham et al. (1995), Wohlin et al. (2000), Kitchenham et al. (2002), Jedlitschka (2005) e baseada na experiência relatada em Silva Filho et al. (2005).

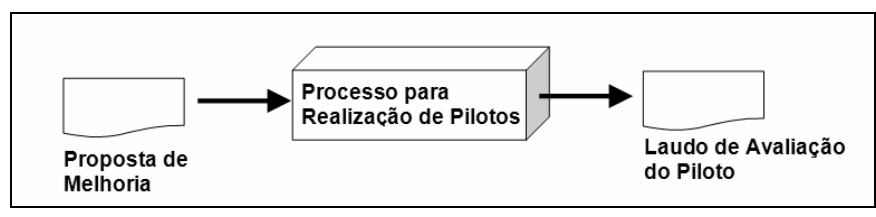

Figura 1 - Visão macro do processo

Para início do processo a proposta de melhoria a ser testada precisa conter os objetivos de melhoria, os resultados esperados com a aplicação das ações de melhoria no(s) processo(s), a descrição detalhada das ações de melhoria e o contexto as quais as melhorias se aplicam (tipo, tamanho e duração de projeto, paradigma etc.). Uma vez que a proposta atenda a estes critérios, o processo é iniciado e a primeira atividade a ser executada é a que trata da definição do piloto a ser realizado.

O processo é finalizado quando todos os projetos-piloto escalados para testar a proposta são concluídos e os dados coletados são compilados através de uma análise quantitativa seguida de uma análise qualitativa. No final do processo, o laudo de avaliação do piloto é emitido contendo os dados consolidados, as ponderações realizadas durante a análise e a informação referente ao atendimento ou não dos resultados às expectativas.

O modelo do processo que constitui o arcabouço envolve a participação dos seguintes papeis:

- Grupo de Processo: Pessoas responsáveis pela definição e melhoria dos processos e ativos de processo da organização;

- Gerente de Projeto: Responsável pela condução das atividades dos projetos desenvolvidos pela organização; e

- Equipe do Projeto: Pessoas que participam do projeto. Dentre eles: analistas, testadores, programadores, arquitetos de software e projetistas.

As atividades do modelo de processo foram agrupadas em seis macroatividades:

\section{A. Definir piloto}

A primeira macroatividade engloba a identificação do piloto, descrição das melhorias a serem avaliadas, definição de um plano de medição e declaração das expectativas para o projeto piloto. Durante a identificação do piloto, o grupo de processo deve procurar analisar a abrangência da proposta e verificar a dificuldade para avaliar os efeitos desejados. Quanto mais difícil de observar os efeitos, maior deve ser o número de projetos que deverão ser associados ao piloto. 
A descrição das ações a serem testadas no processo deve ser realizada tomando como referência o processo ao qual a proposta se aplica. Estas melhorias podem consistir na alteração de atividades (inclusão, alteração, exclusão ou reordenação), utilização de uma nova ferramenta de apoio, utilização de novos métodos e/ou técnicas na execução de determinadas atividades, alteração ou inserção de novos modelos e diretrizes. Deve-se ter atenção para aquelas ações independentes que podem ter influência mútua, principalmente quando a proposta visa atender mais de um objetivo de melhoria. As ações que se influenciam mutuamente podem dificultar a observação dos reais efeitos que cada uma provocou no processo.

A partir da definição dos objetivos, através do método GQM, é possível identificar quais as questões e métricas devem ser utilizadas para mensurar os efeitos das alterações do processo [SOLINGEN e BERGHOUT 1999].

A declaração das expectativas deve ser expressiva e ao mesmo tempo exeqüível. Expectativas muito altas são difíceis de serem satisfeitas, e isso pode implicar a avaliação quantitativa negativa e ocultar a efetividade de uma proposta. Por outro lado, expectativas muito baixas não são úteis, pois, de acordo com o tipo de melhoria, o efeito positivo observado quantitativamente pode não ser expressivo o suficiente para justificar a institucionalização das melhorias. Organizações imaturas têm dificuldade para declarar expectativas, por mais simples que sejam. Sugerem-se como importantes fontes: benchmarking, análise de dados históricos e opinião de especialistas.

\section{B. Definir diretrizes para realização do piloto}

Esta macroatividade resultará nas diretrizes que apoiaram a seleção do projeto a ser utilizado como piloto para avaliar a melhoria. Também está inclusa a atividade que apoiará a identificação dos fatores que podem exercer alguma influência durante a execução do projeto de forma a dificultar a avaliação quantitativa e/ou qualitativa das melhorias a serem testadas.

Há casos em que a avaliação somente poderá ser realizada se o projeto apresentar uma determinada característica. Por exemplo: se no processo de desenvolvimento de software foi inserida uma atividade que se aplica apenas ao desenvolvimento de softwares para web, o projeto-piloto deverá possuir tal característica. Da mesma forma, caso a organização queira avaliar o desempenho do grupo de trabalho com o uso de uma nova tecnologia, é necessário selecionar participantes que tenham o mesmo nível de conhecimento da tecnologia anterior.

\section{Realizar preparação para executar o projeto-piloto}

O grupo de processo encaminha o documento de diretrizes para realização do piloto para $\mathrm{o}$ gerente do projeto. $\mathrm{O}$ gerente do projeto trata de associar o seu projeto ao piloto e utiliza as diretrizes para planejar o processo, planejar o projeto, treinar a equipe, estabelecer o plano de medição para o projeto-piloto e preparar o ambiente para execução. É importante ressaltar que estas tarefas associadas à preparação também podem pertencer ao escopo de outros processos e/ou áreas de processo (vide Figura 2). 


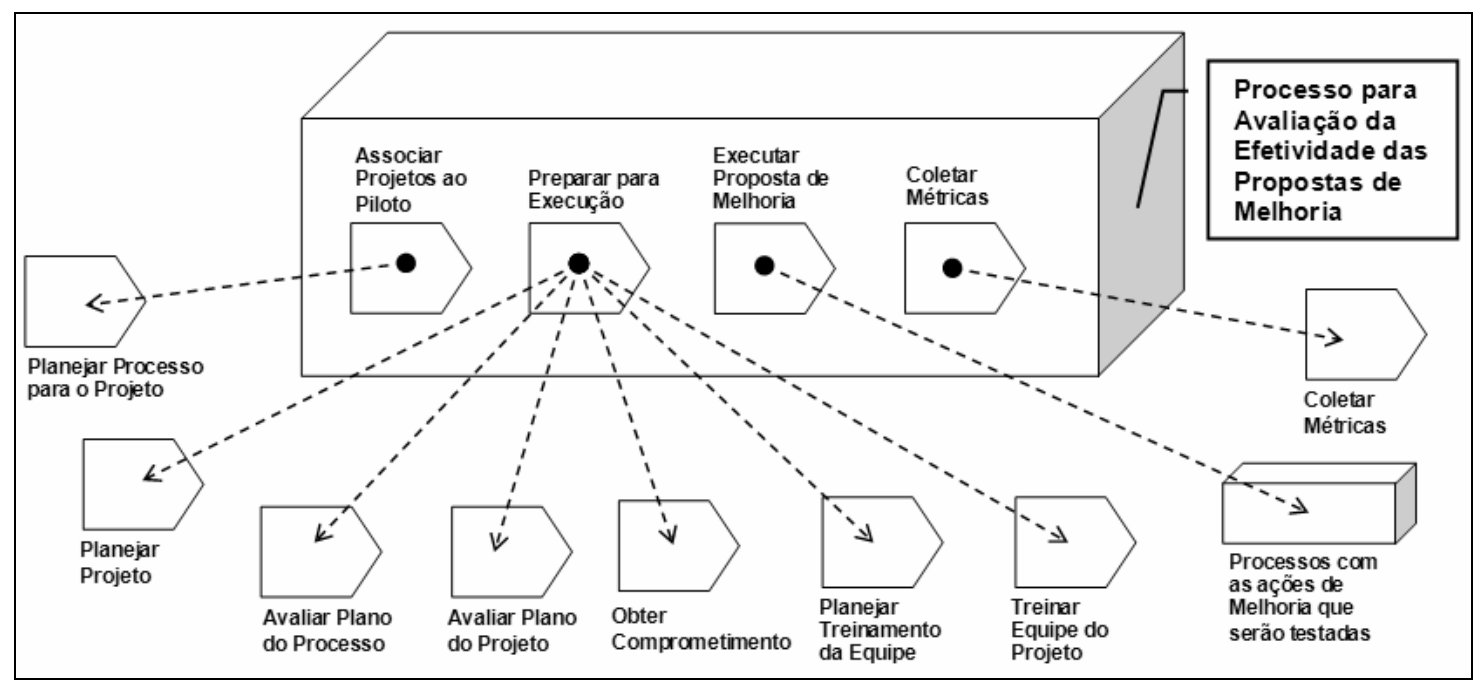

Figura 2 - Interação do elemento processo com outros processos das organizações

\section{Monitorar projetos-piloto}

Dependendo do tipo de proposta que está sendo testada, pode não ser necessário finalizar os projetos para realizar a análise dos dados coletados durante a execução das partes que foram alteradas no processo. Ao realizar uma monitoração freqüente, além de identificar a partir de qual momento o projeto-piloto pode ser dado como finalizado, a monitoração pode ser um instrumento que permite com que o grupo de processo possa observar de perto o momento em que a ação de melhoria irá ser executada e, assim, coletar outras informações que irão apoiar a análise. Contudo, recomenda-se realizar a monitoração de forma não intrusiva para diminuir potenciais influências associadas à situação "sob avaliação" durante a execução do projeto.

Enquanto o grupo de processo monitora a execução das propostas que estão sendo testadas, a equipe do projeto também realiza o registro da medição para as métricas do projeto-piloto.

Antes das atividades de análise dos dados, quando o projeto-piloto for considerado finalizado, realiza-se uma reunião para capturar a avaliação da proposta sob a perspectiva dos participantes. Da mesma forma que, durante a execução de experimentos, o feedback dos participantes contribui com a validação dos dados, na verificação da conformidade do processo, na identificação de problemas de condução e no entendimento da percepção dos participantes [KARAHASANOVIC et al. 2005], recomenda-se que a análise qualitativa também faça uso deste recurso.

O registro das dificuldades para executar a proposta e da resistência de alguns participantes da equipe, dentre outros aspectos, podem auxiliar de maneira significativa a análise qualitativa dos efeitos observados.

\section{E. Analisar os resultados dos projetos-piloto}

Os dados coletados são computados e comparados às expectativas declaradas na proposta de melhoria. O resultado da análise quantitativa poderá atender integralmente, parcialmente ou não atender as expectativas. Quando mais de um projeto está associado ao piloto, é possível obter resultados conflitantes, ou seja, o atendimento parcial às expectativas. Nestes casos, a análise qualitativa deve ser ainda mais criteriosa. O grupo 
de processo deve examinar com cuidado os fatores de influência, o impacto de cada um nos projetos e considerar os dados coletados durante a monitoração e na reunião de feedback para apoiar a interpretação dos resultados.

\section{F. Visualizar Resultados}

Todos os dados referentes ao piloto são concatenados e formatados em um único relatório que ao final emite o parecer a respeito da avaliação da efetividade da proposta de melhoria.

\subsection{O Elemento Ferramenta}

O elemento ferramenta automatiza a execução do processo e se integra à Ambientes de Desenvolvimento de Software ${ }^{3}$ permitindo uma interação transparente com outros processos e ferramentas que compõe o ambiente da organização. A ferramenta não dá suporte a todas as atividades do processo. Foram selecionadas apenas atividades onde a automação traria mais benefícios, ou seja, aquelas que demandariam de mais esforço para serem executadas. A Figura 3 ilustra as atividades do processo que foram contempladas na ferramenta Pilot.

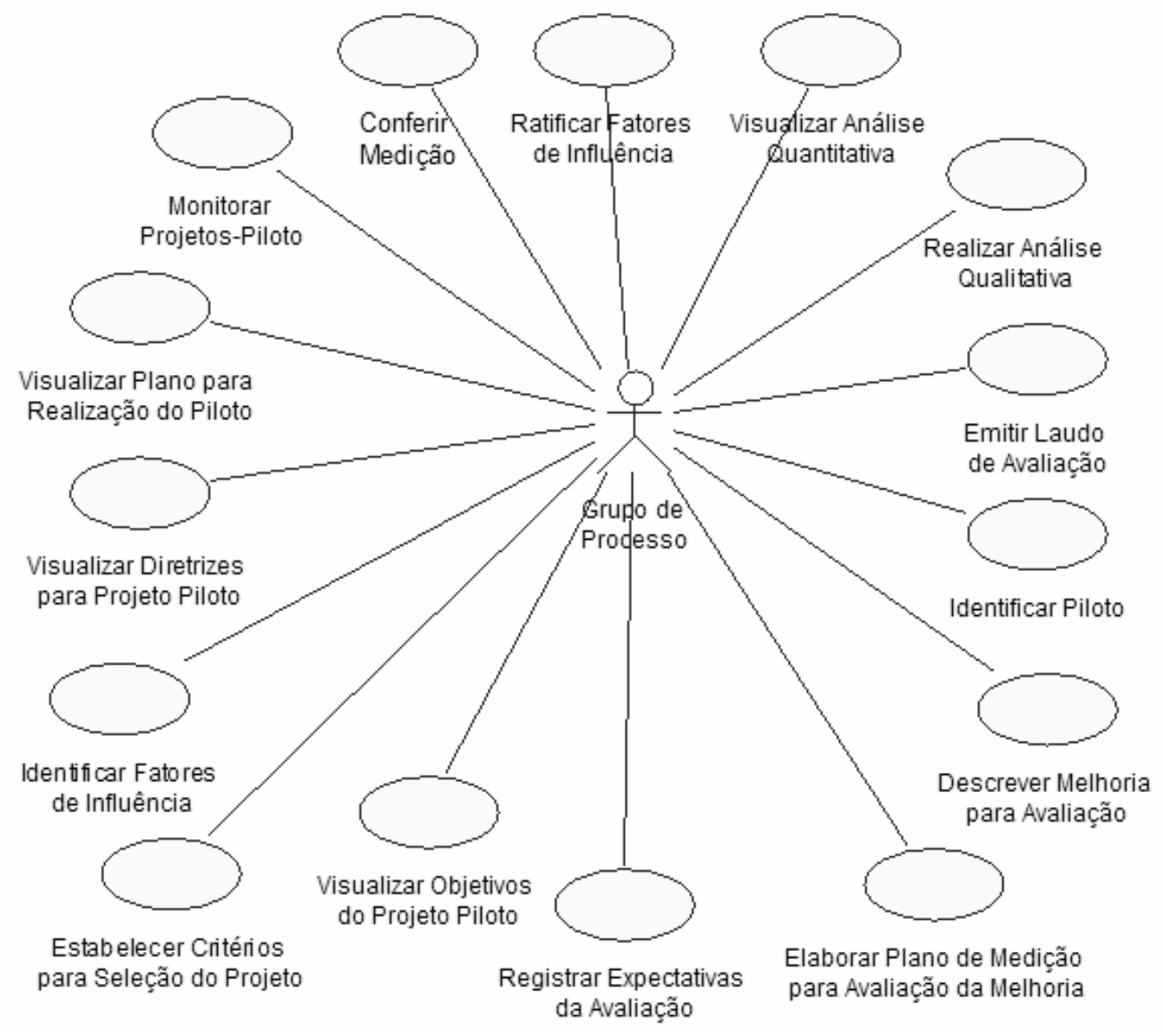

Figura 3 - Diagrama de casos de uso com as funcionalidades da Pilot

\footnotetext{
${ }^{3}$ A integração da ferramenta que compõe o arcabouço foi realizada com a Estação TABA, ADS proposto inicialmente por Rocha et al. (1990) que está em constante evolução e vem sendo amplamente utilizado na indústria de software no Brasil.
} 
O estudo apresentado em Silva Filho et al. (2005) apresentou um custo de aproximadamente $3 \%$ do projeto que foi utilizado como piloto. Tendo em vista as atividades que foram realizadas manualmente, observa-se que com o apoio ferramental o esforço poderia ser reduzido significativamente, propiciando inclusive a associação de mais de um projeto ao mesmo piloto sem que isso tenha impacto significativo nos custos do estudo.

A ferramenta também serve de elemento facilitador na utilização do guia, visto que o conhecimento requerido para executar cada atividade do processo pode ser acessado através de apenas um clique.

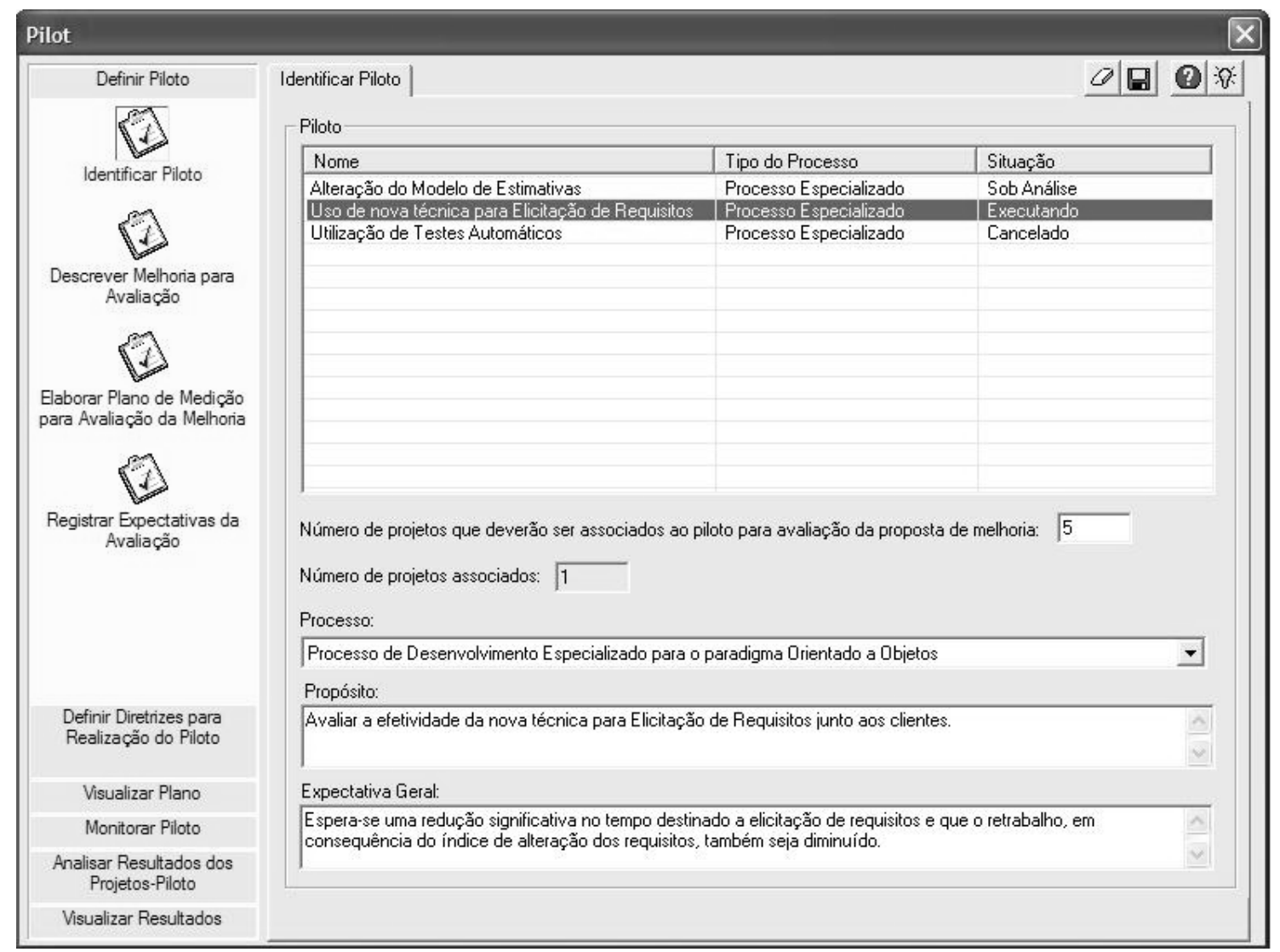

Figura 4 - Tela inicial da ferramenta que integra o arcabouço

\subsection{O Elemento Guia}

O objetivo do guia é fornecer o conhecimento necessário para compreensão dos elementos do arcabouço e habilitar os usuários a utilizá-lo. Ele está dividido em três seções: a primeira apresenta uma visão geral e informa como o guia deve ser utilizado; a segunda parte do guia contém um roteiro para aplicação do processo através da ferramenta, partindo de um exemplo real e uso de um vocabulário adequado ao perfil de grupo de processo; e a última parte contém diretrizes e informações que apóiam o usuário a tomar decisões e sensibilizá-lo ao contexto da avaliação para que ele seja capaz de capturar informações importantes ao longo da realização do piloto e utilizá-las na análise qualitativa.

Dentre as questões centrais abordadas pelo do guia destacam-se: 
a) Número de projetos-piloto. Fornece subsídios para que o usuário possa identificar quantos projetos-pilotos são necessários para avaliar uma proposta de melhoria, com base no risco, amplitude da proposta, urgência, disponibilidade de recursos e impacto na organização.

b) O tratamento da validade. A questão da validade está relacionada aos conceitos de validade interna e externa abordados por Wohlin et al. (2000), que trata, respectivamente, da corretude dos dados coletados e do significado destes dados com relação ao contexto geral (potencial de generalização). Considerar contexto, critérios de seleção do projeto a ser utilizado como piloto, seleção dos indivíduos, necessidade de treinamento e fatores que podem influenciar a avaliação dos resultados é indispensável.

c) Diretrizes para seleção de métricas. O GQM facilita a identificação de métricas para mensuração dos efeitos da mudança, entretanto também é necessário mensurar os fatores que podem influenciar nos efeitos que se pretende observar. Por outro lado, mensurar todos os fatores pode comprometer a medição e implicar no aumento do consumo de recursos do projeto.

d) Identificação dos fatores de influência. $\mathrm{O}$ ambiente industrial está sujeito a intempéries de todos os tipos. É importante concentrar-se nos riscos que podem afetar diretamente a proposta. Tão importante quanto prever, é identificar quando o evento ocorreu. Pois mesmo que não seja possível remediar os efeitos da ocorrência do risco, a análise qualitativa deve levá-los em consideração durante a confecção do laudo.

e) Realizar reunião de avaliação. Para conduzir reuniões com alto grau de aproveitamento podem ser aplicadas técnicas específicas, conforme o objetivo da reunião. O guia apresenta algumas alternativas para dinamizar e potencializar a coleta do feedback dos participantes dos projetos-piloto.

f) Análise qualitativa. A análise qualitativa envolve muita subjetividade. É importante que o grupo de processo tenha sensibilidade ao avaliar o contexto ao qual cada projeto-piloto foi realizado e cruzar estas informações com os dados quantitativos. $\mathrm{O}$ estudo da relação causa-efeito requer atenção, percepção e capacidade para relacionar aspectos que, aparentemente, não possuem relação.

\section{Conclusão}

Este trabalho apresentou um arcabouço para avaliar a efetividade das propostas de melhoria de processos de software no ambiente industrial. Os três elementos que compõe o arcabouço viabilizam a aplicação de uma abordagem experimental para a realização de pilotos, através da sistematização, fornecimento de conhecimento e automação das atividades.

O uso do arcabouço tende a potencializar a realização das avaliações das propostas de melhoria, tornando-as mais acuradas sem que isso implique custos ou aumento do esforço. Além disso, as organizações que adotam e/ou têm interesse em obter certificações em modelos de qualidade poderão contar com a ampla cobertura das práticas e o fornecimento de indicadores relacionados à avaliação de propostas de melhorias e realização de pilotos sugeridos pelos modelos mais difundidos.

O arcabouço já está integralmente disponível para as organizações que utilizam a Estação TABA. 


\section{Referências}

AHMED-NACER, Mohamed. Evolution of Software Processes and of their Models: A Multiple Strategy Approach. Journal of Research and Practice in Information Technology, Vol. 36, Sydney, p. 9-22. Feb. 2004.

BASILI, V. R.; CALDIERA, G.; ROMBACH, H. D. The Experience Factory. In: MARCINIAK, John J.. (Ed.). Encyclopedia of Software Engineering. New York: John Wiley \& Sons, 1994. p. 469-476.

BEITZ, A., WIECZOREK, I. Applying Benchmarking to Learn from Best Practices, Lecture Notes in Computer Science, Vol. 1840, p. 59-72, Jan. 2000.

CARD, D.; ZUBROW, D. Benchmarking software organizations. IEEE Software, Vol.18, no.5, p.16-17, Sep.-Oct. 2001.

CHRISSIS, Mary Beth; KONRAD, Mike; SHRUM, Sandy. CMMI®: Guidelines for Process Integration and Product Improvement. Boston: Addison Wesley, 2003. 688 p. (The SEI Series in Software Engineering).

DYBA, T.; KITCHENHAM, B.A.; JORGENSEN, M. Evidence-based Software Engineering for Practitioners. IEEE Software, Vol. 22, no.1, p. 58-65, Jan.-Feb. 2005 .

FOWLER, P., RIFKIN, S., Software Engineering Process Group Guide, Technical Report CMU/SEI-90-TR-24 ADA235784, Carnegie Mellon University, Software Engineering Institute, Pittsburgh. 1990.

FENTON, N.; PFLEEGER, S.L.; GLASS, R.L. Science and substance: a challenge to software engineers, IEEE Software, Vol. 11, Issue 4, p. 86-95, Jul. 1994.

HÖST, M.; JOHANSSON, C. Evaluation of code review methods through interviews and experimentation. Journal of Systems and Software, Vol. 52, Issues 2-3, p.113120. 1 Jun. 2000.

HÖST, Martin et al. Exploring bottlenecks in market-driven requirements management processes with discrete event simulation. Journal of Systems and Software, Vol. 59, Issue 3, p. 323-332. 15 Dez 2001.

ISO. ISO/IEC 15504-4 Information Technology - Process Assessment, Part 4: Guidance on use for Process Improvement and Process Capability Determination (2004)

KARAHASANOVIC, Amela et al. Collecting Feedback During Software Engineering Experiment, Empirical Software Engineering, Vol. 10, Issue 2, p. 113-147. Apr 2005.

KELlEY, D. L., MORATH, P. How Do You Know The Change Worked? Quality Progress, American Society for Quality, Vol. 34, p. 68-74, Jul. 2001.

KELLNER, M. I.; MADACHY, R. J; RAFFO, D. M. Software process simulation modeling: Why? What? How? Journal of Systems and Software, Vol. 46, Issues 2-3, p. 91-105. 15 Apr. 1999.

KITCHENHAM, B.; PICKARD, L.; PFLEEGER, S.L. Case studies for method and tool evaluation. IEEE Software, Vol. 12, Issue 4, p. 52-62, Jul. 1995 
KITCHENHAM, B.A.; PFLEEGER, S.L.; PICKARD, L.M.; JONES, P.W.; HOAGLIN, D.C.; EMAM, K. EL; ROSENBERG, J. Preliminary Guidelines for Empirical Research in Software Engineering. IEEE Transactions on Software Engineering, Vol. 28, no. 8, p. 721-734, August 2002.

MCFEELEY, Bob. IDEAL ${ }^{\text {SM }}$ A User's Guide for Software Process Improvement", Handbook CMU/SEI-96-HB-001, Software Engineering Institute, Feb. 1996.

MPS.BR - Melhoria de Processo do Software Brasileiro, Guia Geral (v. 1.0) . Sociedade SOFTEX, Brasil. (2005)

MÜNCH, J.; ARMBRUST, O. Using Empirical Knowledge from Replicated Experiments for Software Process Simulation: A Practical Example. In: Proceedings of the 2003 International Symposium on Empirical Software Engineering (ISESE'03), IEEE Computer Society, 30, p.18-27. Sept.-1 Oct. 2003.

MÜNCH, J.; PFAHL, D.; RUS, I. Virtual Software Engineering Laboratories in Support of Trade-off Analyses. Software Quality Journal, Vol.13, Issue 4, p. 407-428. Dec. 2005.

PAULK, M. et al. Capability maturity model, version 1.1, IEEE Software, vol.10, no.4, p.18-27, Jul 1993.

PFAHL, D.; BIRK, A. "Using Simulation to Visualise and Analyse Product-Process Dependencies in Software Development Projects", Lecture Notes in Computer Science, Vol. 1840, Jan 2000, Page 88.

PFAHL, D.; LEBSANFT, K. Using simulation to analyse the impact of software requirement volatility on project performance. Information and Software Technology, Vol. 42, Issue 14, Pages 1001-1008. 15 Nov. 2000.

PFAHL, D.; RUHE, G. "IMMoS - A methodology for integrated measurement, modelling, and simulation", Software Process Improvement and Practice, Vol. 7, p. 189-210. Dez 2002.

RAFFO, David. Evaluating the impact of process improvements quantitatively using process modeling, Proceedings of the 1993 conference of the Centre for Advanced Studies on Collaborative research: software engineering - Vol. 1, IBM Press, Toronto, p. 290-313.

ROCHA, A. R. C., AGUIAR, T. C., SOUZA, J. M. Taba: A Heuristic Workstation for Software development. In: Proceedings of COMPEURO 90, Tel Aviv, Israel. May 1990.

SHULL, Forrest et al. Knowledge-Sharing Issues in Experimental Software Engineering, Empirical Software Engineering: An International Journal, Vol. 9, No. 1, pp.111-137. 2004.

SILVA FILHO, R. C., ROCHA, A. R., TRAVASSOS, G. H., Uma Abordagem Experimental para Avaliação da Melhoria de Processos. In: SBQS - Simpósio Brasileiro de Qualidade de Software, 4., 2005, Porto Alegre. Congresso. Porto Alegre: SBC, 2005. p. 395 - 402. Jun. 2005. 
SJØBERG, Dag I. K. et al., Conducting realistic experiments in software engineering, In: Proceedings of the 2002 International Symposium on Empirical Software Engineering, vol., no.pp. 17- 26, Oct. 2002.

SJØBERG, Dag I. K. et al. A Survey of Controlled Experiments in Software Engineering, Software Engineering, IEEE Transactions on, vol.31, no.9pp. 733- 753, Sept. 2005.

SOLINGEN, Rini Van; BERGHOUT, Egon. The Goal/Question/Metric Method: a practical guide for quality improvement of software development. London: McGrawHill, 1999.

TVEDT, J. D., COLlOFELlO, J. S., Evaluating the effectiveness of process improvements on software development cycle time via system dynamics modeling. In: Annual International Computer Software and Application Conference, 19., 1995, Dallas. Proceedings. IEEE Computer Society Press, p. 318-325. 1995.

ZELKOWITZ, M. V., WALLACE, D. R., BINKLEY, D. W., Experimental Validation of New Software Technology, In: Lecture Notes on Empirical Software Engineering, World Scientific Publishing, p. 229-263. 2003.

WALRAD, C.; MOSS, E. Measurement: the key to application development quality, IBM Systems Journal, Vol. 32, N. 3, p. 445-460. set 1993.

WOHLIN, Claes et al. Experimentation in Software Engineering - An Introduction. Massachusets: Kluwer Academic Publishers, 2000. 204 p. (The Kluwer International Series in Software Engineering). 